\title{
On the influence of monochromator thermal deformations on X-ray focusing
}

\author{
M.A. Antimonov ${ }^{1,3}$, A.M. Khounsary ${ }^{1,2, a}$, A. Sandy ${ }^{3}$, S. Narayanyan ${ }^{3}$, and G. Navrotski ${ }^{3}$ \\ ${ }^{1}$ Department of Mechanical and Industrial Engineering, University of Illinois at Chicago, Chicago, 60607, USA \\ ${ }^{2}$ Department of Physics, Illinois Institute of Technology, Chicago, IL 60616, USA \\ ${ }^{3}$ Argonne National Laboratory, Argonne, 60439, USA
}

A cooled double crystal monochromator system is used on many high heat load X-ray synchrotron radiation beamlines in order to select, by diffraction, a narrow spectrum of the beam. The wavelength of the diffracted beam is tuned by changing the angle of incidence, per the Bragg equation. Thermal deformation of the first crystal monochromator - and the potential loss of beam brilliance - is often a main concern. However, if downstream beam focusing is planned, the lensing effect of the monochromator must be considered even if thermal deformations are small enough to have any imperceptible impact on intensity. In this paper we report on recent experiments at an Advanced Photon Source (APS) beamline that uses focuses compound diffractive lenses downstream of an X-ray monochromator system. Increasing the X-ray beam power by increasing the storage ring current from $100 \mathrm{~mA}$ to $130 \mathrm{~mA}$ resulted in the doubling of the focal distance, to some location outside the experimental hutch. We show quantitatively that this is be due to the well-known lensing effect of a distorted monochromator that results in the creation of a virtual source, at some distance downstream of the actual source. An analysis of the defocusing and options to mitigate are provided.

Keywords: Synchrotron X-ray Radiation, Focusing, Monochromator, Thermal Deformations, Cooling

a) Corresponding author: amk@iit.edu.

\section{INTRODUCTION}

The thermal load of a polychromatic synchrotron X-ray beam incident on the first crystal of a double crystal monochromator (DCM) usually results in the development of thermal deformations - thermal bumps - on the crystal surface.

This deformation perturbs the crystal lattice under the beam footprint leading to a broadening of the rocking curve of the $\mathrm{DCM}^{1}$. This broadening - and the resulting intensity loss from a DCM - may or may not be significant depending on the severity of the deformation: if the root mean square (rms) of its first derivative along the beam (i.e., the rms tangential slope error) is smaller than the Darwin width of the crystal, the broadening of the rocking curve and loss of intensity would not be significant. Yet a monochromator with its low level of thermally induced deformation, acting like a convex mirror, can perceptibly increase the diffracted beam divergence. This 'lensing' effect of a deformed monochromator has previously been studied $^{2,3,4}$ and methods to take advantage of it $^{2}$ and mitigate $i^{3}$ have been proposed. Measurement of the thermal deformation ${ }^{5}$ and its impact on beam wavefront has also recently been studies ${ }^{6}$. The additional divergence due to the lensing of the monochromator leads to an apparent or virtual x-ray source that is closer to the DCM than the real source and thus 
13 focusing optics will focus the beam beyond the expected location. If the optics has an adjustable focal length (as is the case 14 with adaptive or bendable mirrors ${ }^{7,8}$, transfocators ${ }^{9}$, or saw-tooth refractive lenses ${ }^{10}$ ), the change in focal distance can be 15 reversed: the beam can be re-focused at the desired position even if the beam power (and thus thermal distortion) varies. 16 However, if the focusing optic has a fixed focal length, or if the change in thermal load / thermal deformation is faster than 17 optics can be adjusted, thermal deformations can lead to a significant shift in focal distance. This was observed at Advanced 18 Photon Source 8-ID beamline during a test in which the storage ring beam current was increased to increase the thermal load 19 on the optical systems throughout APS. What is significant is that even modest thermal deformation levels in the crystal monochromator - too small to impact diffracted intensity - can shift the focus significantly. The challenge for the beamline was to analyze the situation and develop options to minimize focal distance shifts.

Thermal deformations of monochromators on high heat load X-ray beamlines has been one of the most widely discussed 23 optics challenge since the emergence third generation $\mathrm{X}$-ray facilities utilizing insertion devices ${ }^{11,12,13}$. To reduce the thermal 24 deformations various solutions, including liquid metal cooling, were proposed ${ }^{11}$. Other techniques such as spreading the 25 beam over larger footprints using inclined surfaces ${ }^{13}$ or asymmetrically cut crystals ${ }^{14}$ were explored. But the most effective technique for mitigating thermal deformation in silicon monochromator is cryogenic cooling where the combination of high thermal conductivity and low thermal expansion of silicon crystal is exploited ${ }^{12}$. In fact, single crystal silicon has a negative but small thermal expansion coefficient below $125 \mathrm{~K}$ which means the thermal deformation under the incident beam footprint can have a concave shape. Utilizing this concave shape to converge the diffracted beam for additional focusing when thermal deformation is present has been recently proposed ${ }^{15}$. This would not apply in the present situation because the Germanium monochromator at Sector 8-ID at the APS does not have negative thermal expansion and in any case is operated at room temperature (water cooled).

The remainder of this paper is organized as follows. First, we describe the experimental set up and the measurement of 34 focal distance at 8-ID beamline as a function of beam power varied by changing the APS storage ring current. We then 35 analyze the thermal deformation of the first germanium monochromator and its impact on the performance of a compound refractive lens (CRL) used downstream of the DCM for X-ray focusing. Third, we demonstrate that the focal distances measured can be predicted analytically as well as numerically. Finally, we propose and discuss possible modifications of the monochromator design to reduce thermal deformation and changes in focal distance.

\section{Beamline and Measurements}

\section{$40 \quad 2.1$ 8-ID beamline description}


A schematic of the APS 8-ID beamline is presented in Figure 1. The X-ray source is a either one or two (tandem) Undulator A (UA) ${ }^{16}$. Optically significant beamline components include a circular pinhole $26 \mathrm{~m}$ downstream of the source, a reflecting silicon mirror and a $250-\mu \mathrm{m}$ thick beryllium window at about $29 \mathrm{~m}$. The diameter of the pinhole is $280 \mu \mathrm{m}$. The incident angle of the beam on the mirror is $0.15^{\circ}$. The mirror acts like a low-pass filter with a cut-off energy of approximately $12 \mathrm{keV}$ transmitting a so-called pink into 8-ID-D hutch.

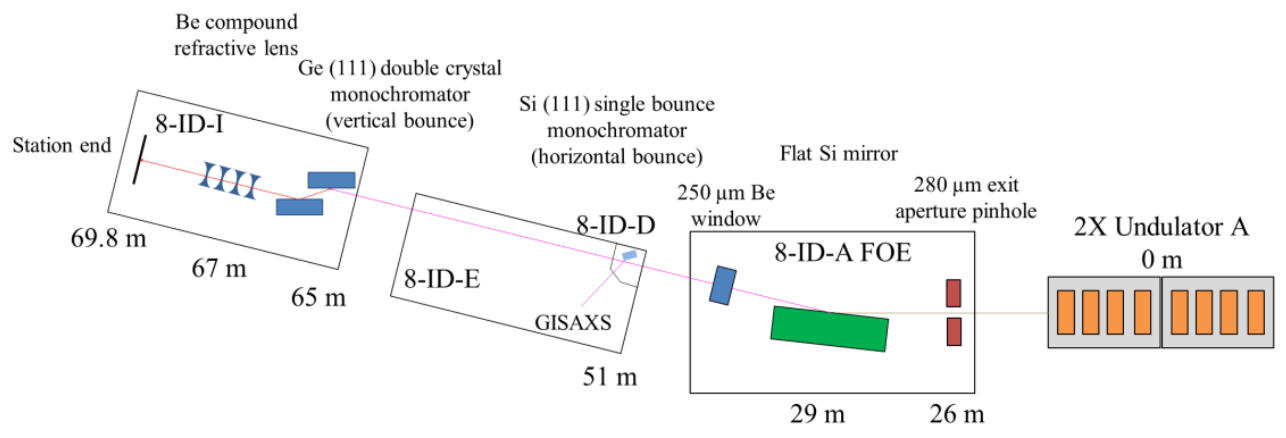

FIG 1. A top-view schematic of the APS 8-ID beamline, except for 8-ID-I box which is a side view for clarity

In 8-ID-D area, the beam is cut in half by a silicon crystal located $51 \mathrm{~m}$ from the source providing a monochromatic beam for experiments at 8-ID-E. The remaining half of the beam proceeds to 8-ID-I hutch where it is monochromatized at 65 m by a Ge(111) vertically diffracting double crystal monochromator. Downstream the monochromator, the beam is focused vertically by a beryllium compound refractive lens (CRL) assembly consisting of ten double-concave parabolic lenses having osculation radius of $200 \mu \mathrm{m}$. Other details of APS 8-ID beamline can be found in the previous work ${ }^{17}$.

\subsection{Experimental measurements of focal distances}

While the normal operation regime of the beamline is $7.35 \mathrm{keV}$ (17.6 mm undulator A gap), some experiments were performed at $8.1 \mathrm{keV}$ (18.6 mm undulator gap). The expected focal distances at these energies are $1.64 \mathrm{~m}$ and $1.99 \mathrm{~m}$ respectively. However, focal distance measurements showed a downstream shift in focus due to thermal deformation of the monochromator. For example, for the case of two UA beams at $8.1 \mathrm{keV}$, the measured the focal distance increased $22 \%$, from $1.99 \mathrm{~m}$ to $2.43 \mathrm{~m}$. Using XOP software ${ }^{18}$, the beam power incident on the first monochromator is estimated to be $1 \mathrm{~W}$ for one UA and $2 \mathrm{~W}$ for two UAs at $100 \mathrm{~mA}$ beam current. Next, the storage ring current was increased from $100 \mathrm{~mA}$ to 130 $\mathrm{mA}$ and the silicon crystal was moved out so the germanium monochromator was heated by the full beam. The intercepted $8.1 \mathrm{keV}$ beam total power by the Ge monochromator was $2.6 \mathrm{~W}$ for one UA and 5.2 for two UA sources.

Beam size at various locations downstream of the CRLs in 8-ID-I hutch was measured and the results are plotted in Figure 2. Since the focus was (unexpectedly) beyond the hutch, the focal length could not be measured directly. Instead, the vertical converging beam profile was measured at several locations downstream of the CRLs. Straight line fits to the data 
yield, via extrapolation, the optic-to-image distance. As shown in the figure, for all the conditions tested, we find an image distance (focal length) significantly larger than that determined from the physical properties of the lens. The only element of

67 the 8-ID beamline optics, which can make such significant impact into the focusing at higher power, is the first crystal in DCM. The analysis of its thermal deformations will be presented in the following section and the modifications of the DCM will be discussed.

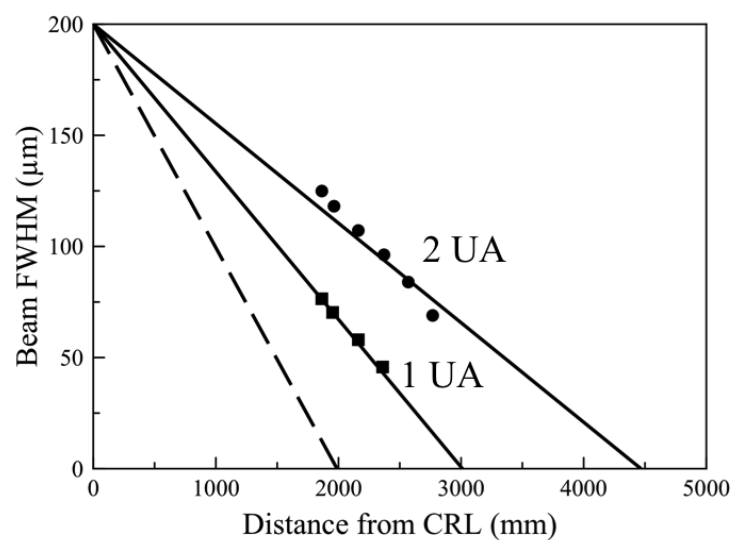

71 FIG 2. Measured vertical beam size (symbols) and the best curve fits (solid lines) through them and extrapolated to the where the respective beam hypothetically focus. Squares denote a single Undulator beam (at $18.6 \mathrm{~mm}$ gap with a first harmonic of $8.1 \mathrm{keV}$ ) and 130 mA storage ring current. Circles are for two Undulator A in tandem. The dashed line represents the ideal vertical beam variation downstream of the CLRs.

\section{Analysis of monochromator thermal deformations}

\section{6}

\subsection{Preliminary discussions}

The germanium monochromator is water-cooled at room temperature. The thermal bump is always convex resulting in a diffracted beam with increased divergence and thus a virtual source closer to the monochromator than the actual source.

a)

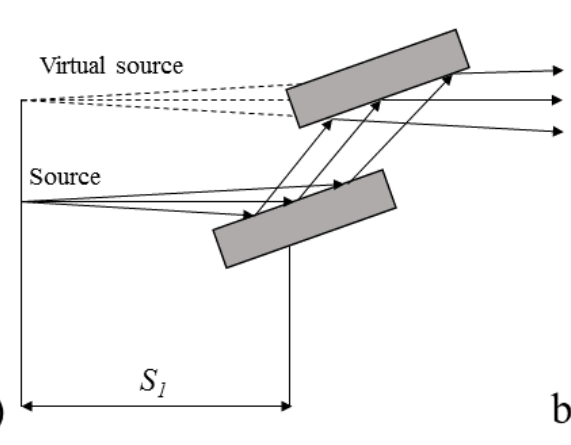

b)

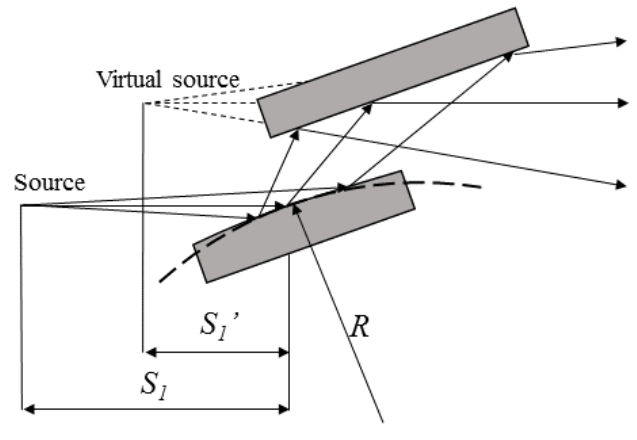

81 the source is at the center of the undulator(s). b) - Thermal deformations add divergence to the rays leading to appearance of a virtual 
To estimate the location of the virtual source, we treat the convexly deformed first monochromator crystal as a tangentially focusing mirror ${ }^{19}$

86 where $S_{1}$ and $S_{1}$ ' are the positive source-to-crystal and crystal-to-virtual source distances, $R$ is the positive radius of the best-

87 fitting cylinder to the convex thermal bump, and $\alpha$ is the incident angle of the central ray. Thus, the distance between the 88 virtual source and the first crystal is given by

undulator source. The virtual source-to-CRL distance is thus $S_{1}{ }^{2}+d$ where $d$ is the distance between the monochromator and the CRLs. The focal distance, $S_{2}$, is then calculated using the thin lens equation

$$
\frac{1}{S_{1}^{\prime}+d}+\frac{1}{S_{2}}=\frac{1}{f}
$$

94 where $f=R_{0} / 2 N \delta$ is the focal length of the CRL consisting of $N$ biconcave lenses with osculating radius $R_{0}$ and $\delta=1-n$ is 95 the decrement of the refractive index $n^{19}$.

These analytical formulations are approximate and in the next section these results are compared with the more precise 97 ray tracing computations carried out for the subject beamline. We then proceed to compute the thermally-induced 98 deformations in the crystal, obtain a fitting radius for the thermal bump (see Fig. 4-b), and use this information to obtain the changes in the focal distance of the CRLs. The case of two tandem undulators (data presented by squares in Fig. 2) is used 100 for this purpose.

101 Note that from the equation (3) the minimum allowable virtual source distance is $4.16 \mathrm{~m}$ if the focusing within $2.8 \mathrm{~m}$ is required when the distance $d$ between monochromator and CRL is $2 \mathrm{~m}$.

\subsection{Ray tracing simulations of the beam profile on the monochromator surface}

Ray-tracing software Zemax ${ }^{20}$ is used to simulate the X-ray undulator beam profile at the first crystal monochromator. The model includes a source, a circular pinhole, and a "detector" at the monochromator location. To simulate the source, the XURGENT program in $\mathrm{XOP}^{18}$ software is used to compute the spatial and angular distributions of the source which were then fitted with Gaussian curves. Spatially, the best fit curves have horizontal and vertical FWHMs of 644 and $28 \mu \mathrm{m}$, respectively. Angularly, the FWHMs are 153 and $96 \mu \mathrm{rad}$. These values were used as input into Zemax source model. The beam cross section at monochromator location is presented in Figure 4. 

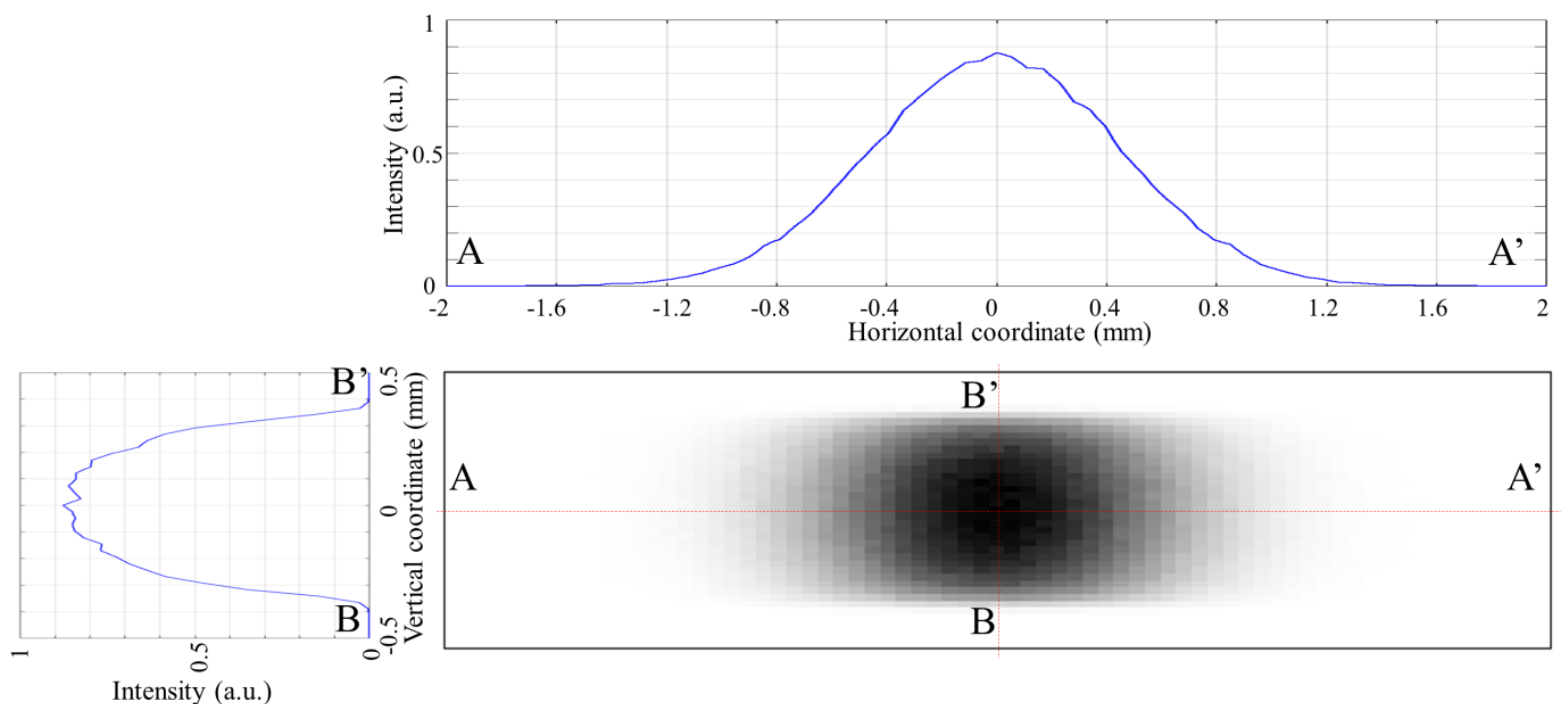

FIG 4. Intensity distribution at the incident X-ray beam at the monochromator location (center image) with the distributions along center

112 lines (above and on the right hand side) also shown. The pixilation of the image is artifact. Full widths at half maximum (FWHM) of the

113 beam at central cross sections are $1.1 \mathrm{~mm}$ (horizontal) and $0.6 \mathrm{~mm}$ (vertical).

\section{$114 \quad 3.3$ Finite element analysis of monochromator thermal deformations}

115 The first germanium monochromator crystal used at APS 8 -ID is $25 \times 34 \times 10.5 \mathrm{~mm}^{3}$ (length $\times$ width $\times$ thickness) in 116 dimensions. It is depicted in the cartoon in Fig. 5 along with incident beam profile and cooling sites used in finite element 117 analysis.

a)

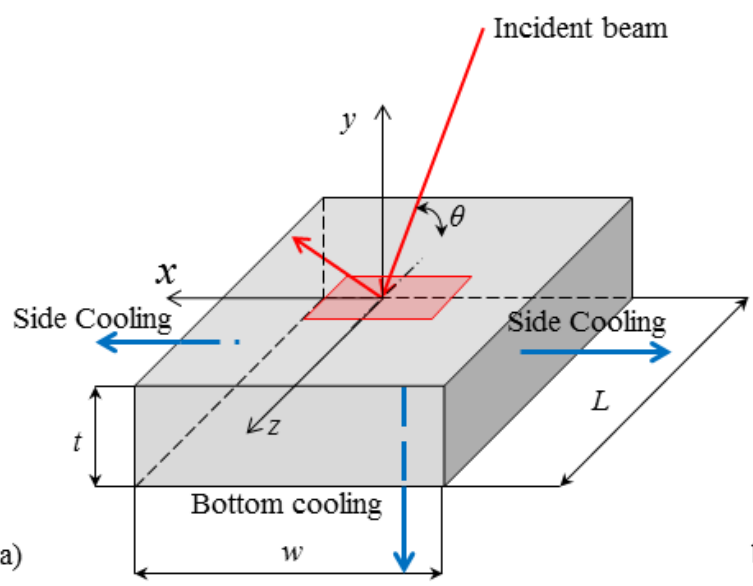

b)

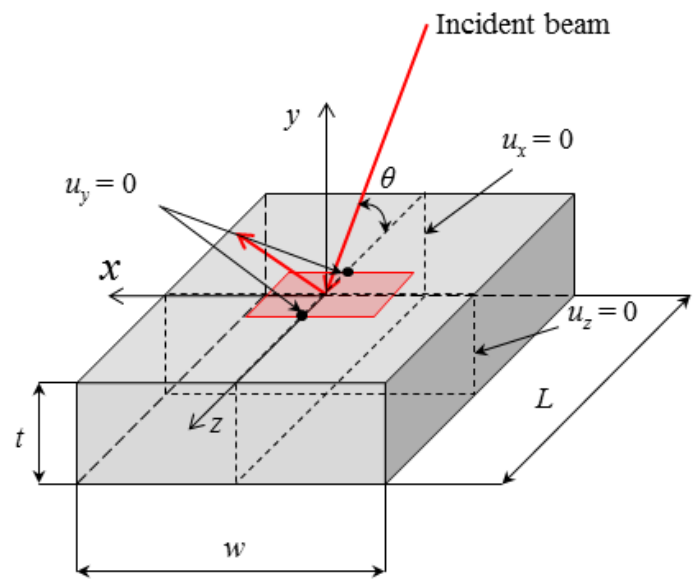

119 FIG 5. Schematic representation thermal (a) and structural (b) boundary conditions of the germanium monochromator, $25 \times 34 \times 10.5$ mm ${ }^{3}$ ( $120 L \times w \times t$ ) in size, used in the finite element analysis. The incident beam footprint (in red) on the top surface is shown. It is analyzed for 121 both bottom and side cooling configurations.

122 Temperature and thermally-induced deformations in the crystal monochromator are obtained using the finite element 123 technique. The thermal and structural boundary conditions are schematically shown in Figure 5-a and 5-b, respectively. In 
124 the analysis, it is assumed that the X-ray beam in absorbed at the surface, a reasonable assumption because of the shallow penetration of subject X-ray beam into germanium. The beam power profile shown in Figure 4 is used in finite element analysis after it is centered and projected onto the surface of the monochromator at $13.55^{\circ}$ (Bragg angle). The total incident beam power is $5.2 \mathrm{~W}$.

Two cooling geometries are considered: bottom cooling (which corresponds to the conditions present at the beamline) and side cooling, which is a possible alternative. At the germanium / cooling block interface, a $25 \mu \mathrm{m}$ thick interstitial silver foil is used to enhance heat transfer (with a conservative value for the contact conductance of $1000 \mathrm{~W} / \mathrm{m}^{2}{ }^{\circ}{ }^{\circ}$ ) ${ }^{21}$. The coolant is water was at $30^{\circ} \mathrm{C}$. All other surfaces are thermally insulated. Temperature dependent material properties are used ${ }^{22}$ in the analysis. Figure 6-a shows the steady temperature distribution in the crystal when bottom cooled.

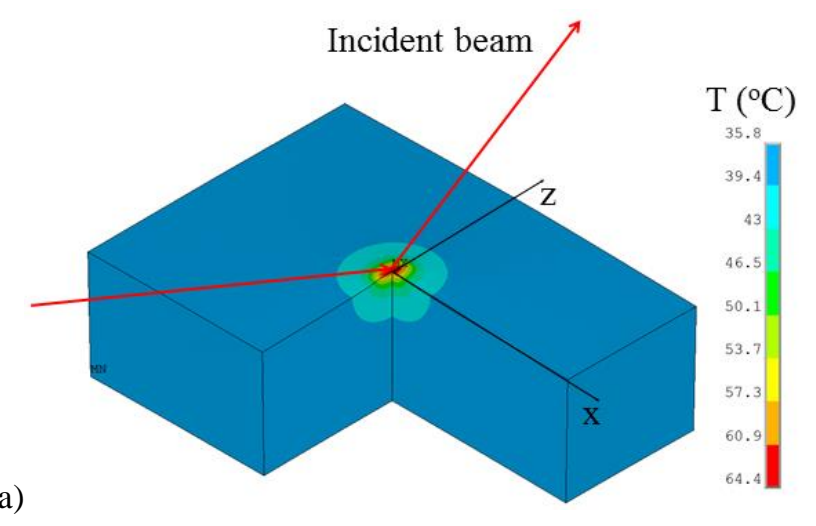

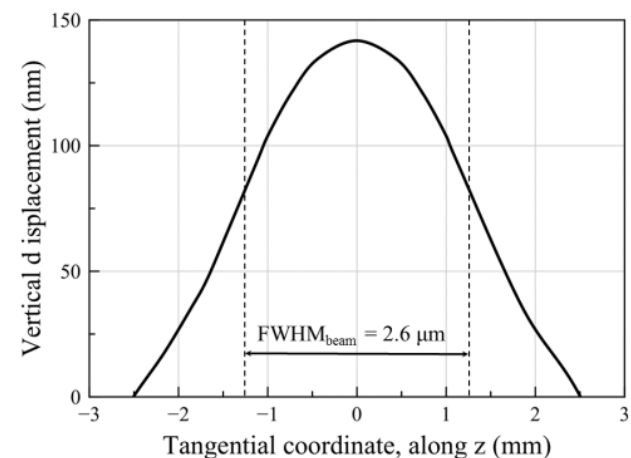

b) a)

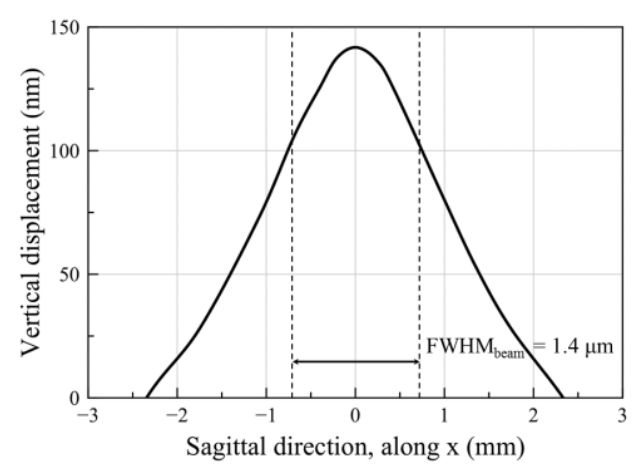

c)

135 FIG 6. First crystal temperature distribution (a) and the surface displacements under the footprint along $\mathrm{z}$ (tangential, b) and $\mathrm{x}$ (sagittal, c) directions. The tangential and sagittal RMS slopes under beam footprint are 64 and $56 \mu$ rad, respectively.

This temperature distribution is used to compute thermal distortions in the crystal. The displacements of the diffracting surface tangentially (along z) and sagittally (along x) directions in case of bottom cooling are shown in Figure 6. For the bottom and side-cooled conditions the tangential radii in the apex of the thermal bumps are found numerically as the inverse of the second derivative of the thermal deformations. They are 12.7 and 12.4 , respectively. These deformations result in 141 virtual sources, respectively, at $1.45 \mathrm{~m}$ and $1.42 \mathrm{~m}$ upstream of the monochromator. In the absence of thermal deformation, $R$ 
142 is infinity in Eq. 2, the number of lenses to focus the beam at $S_{2}=2.0 \mathrm{~m}$ was estimated at $N=10$. When monochromator

143 distortions are present, this lens system, according to Eqns. 2 and 3, will focus the 8.1-keV photon energy at $4.35 \mathrm{~m}$ and 4.4

$144 \mathrm{~m}$ for the bottom and side-cooled conditions. $N=16$ lenses are required to focus the beam at the desired 2-m distance 145 downstream the CRL.

146 The 4.35-m focal distance for bottom cooled monochromator is far from the 2-m value intended, but is in good 147 agreement with the $4.4 \mathrm{~m}$ focal distance predicted from the extrapolation of measured data in Figure 2 . The analysis results 148 for bottom and side cooling shows the same results. Therefore from here on we shall next discuss bottom cooling results 149 only.

Given the strong dependence of the focal distance on monochromator deformation and in turn on thermal loading which 151 can vary during an experiment, it is desirable to investigate options to reduce thermal deformations in the crystal. These 152 include geometry and cooling optimizations, use of a different crystal material with more favorable thermal / structural 153 properties, use of asymmetric crystals, or a combination of the above.

\subsection{Measures to improve the monochromator performance}

\subsubsection{Optimization of crystal geometry}

As a first step in the optimization process, thermal deformations in the first crystal (set to diffract $8.1 \mathrm{keV}$ photons) are computed using thermal / structural finite element analysis. The incident beam is from two in-tandem undulators. The monochromator geometry is varied in the optimization process and for each geometry the apex curvature is calculated. This curvature value is substituted into the equation (2) to calculate the virtual-source position. The optimal monochromator geometries should have sufficiently small deformation resulting in the virtual-source position being not closer than $4.16 \mathrm{~m}$ to parameters of width, length, and thickness, one at a time is varied.

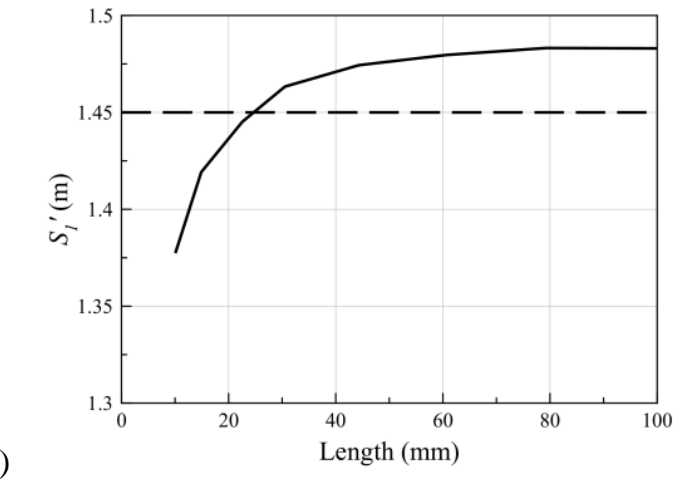

a)

164 b)

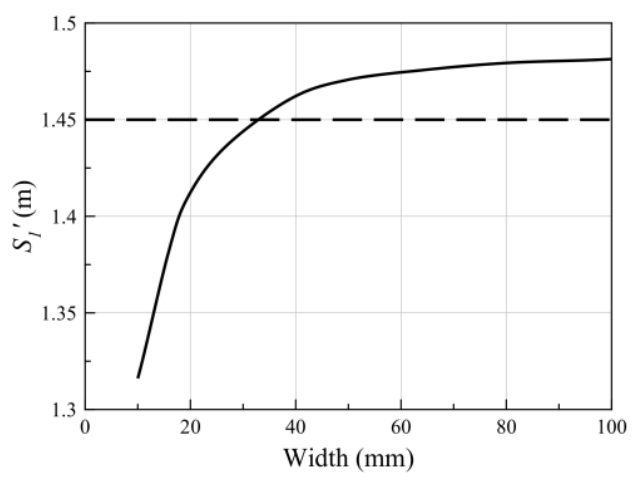




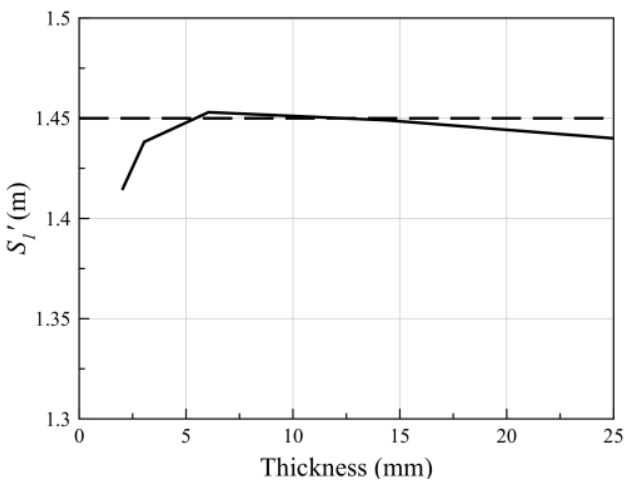

166 FIG 7. Distance between virtual source and monochromator $S_{1}$ ' versus crystal dimensions. Length (a) or width (b) or thickness (c) of the crystal dimensions is changed while the others are held at their original dimensions of $25 \times 34 \times 10.5 \mathrm{~mm}^{3}$ (length $\times$ width $\times$ thickness). Dashed line corresponds to the original $S_{I}$ '. The object is to maximize the virtual-source distance $S_{1}$ ' and bring it closer to the real-source distance $S_{1}$. The minimum acceptable value for $S_{1}$, allowing to focus properly with the existing optics, is $4.16 \mathrm{~m}$.

The results of calculations show that the thermal deformations increase if the distance between cooling surfaces and the heated surface is increased. The increase in heated zone temperature accompanies a decrease in the thermal conductivity of

172 germanium and an increase in its thermal expansion coefficient leading to higher thermal gradients under the footprint and 173 increased thermal strains. This effect explains the existence of the thickness where the virtual source distance is maximum 174 (see Figure 7-c).

175 The finite element results presented show that the thermal deformations cannot be suppressed to an acceptable level by 176 varying of the crystal dimensions. In addition, side cooling of the monochromator does not also provide any advantage 177 because thermal deformation is due to thickening rather than bending of the crystal, which could be reduced by side cooling. 178 Other monochromator improvement options are discussed next.

\subsubsection{Other options to reduce detrimental thermal deformations}

Thermal deformations might be reduced if the existing symmetric water-cooled germanium monochromator crystal is replaced with:

1) Asymmetrically cut germanium crystal;

2) Cryogenically cooled germanium crystal;

3) Silicon crystals.

The dependence of the virtual source distance on the asymmetry cut angle is presented in Figure 8. 


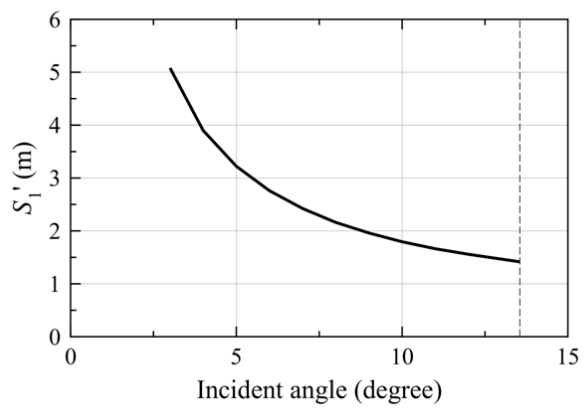

FIG 8. Dependence of the virtual-source distance on the incident angle, which is a difference between Bragg angle and cut angle. Vertical dashed line corresponds to the symmetrically cut crystal, the incident angle equals the Bragg angle $13.55^{\circ}$.

Increasing the crystal asymmetry angle results in elongated footprint and thus decreased incident heat flux and thermal deformation. However, at smaller incident angles, the virtual-source position is more sensitive to surface non-flatness (see 191 equation (2)). Thus, the allowable virtual-source position may be achieved only at asymmetry angle about $10^{\circ}$. $\mathrm{m}$, compared with $72 \mathrm{~m}$ for a water-cooled silicon monochromator. The virtual source distances are then $13.7 \mathrm{~m}$ and $7.8 \mathrm{~m}$, respectively, with expected focal distances of $2.2 \mathrm{~m}$ and $2.4 \mathrm{~m}$ which both are close to the desired $2.0 \mathrm{~m}$ design value. This analysis shows that the monochromator deformations are the least sensitive in case when a silicon monochromator with cryogenic cooling is used. The expected enlargement of the focal distance is $20 \mathrm{~mm}, 10 \%$ of initial focal distance.

\section{RAY-TRACING SIMULATION OF THE EXPECTED FOCUSING OF THE X-RAY BEAM} footprint was used to analytically estimate the image distance produced by the distorted first crystal monochromator. A more detailed representation can be obtained by ray-tracing analysis. This was performed incorporating beamline specifications in

Zemax. The source was simulated by a beam with Gaussian spatial and angular distributions. Rays with 8.1-keV photon energy were used in the analysis. The spatial standard deviations of $274 \mu \mathrm{m}$ (horizontal) and $11 \mu \mathrm{m}$ (vertical) and angular standard deviations of $12.6 \mu \mathrm{rad}$ (horizontal) and $6.69 \mu \mathrm{rad}$ (vertical) ${ }^{16}$ were used. No filters (the high-pass beryllium window and the low-pass mirror) were considered in the ray-tracing analysis because they transmit $8.1-\mathrm{keV}$ energy without attenuation. A $280-\mu \mathrm{m}$ pinhole placed 26.24-m downstream the source and the double crystal monochromator 38.56-m downstream of the pinhole were incorporated. The monochromator was modeled as two surfaces reflecting all incident rays. The profile of the first surface corresponding to the thermally deformed monochromator was imported from the finite element solution. The second surface is assumed flat. The compound refractive lens is placed $2 \mathrm{~m}$ downstream of the double crystal 
monochromator. It consists of 10 beryllium double concave lenses with parabolic cylindrical profile. The osculating radii of the lenses are $200 \mu \mathrm{m}$. The focus was determined by searching for the beam cross-section where the peak intensity of the

211 focused beam was maximum.

212 The profiles of the focused beams for the above four conditions are presented in Figure 9. Corresponding focal distances 213 are listed in Table 1.

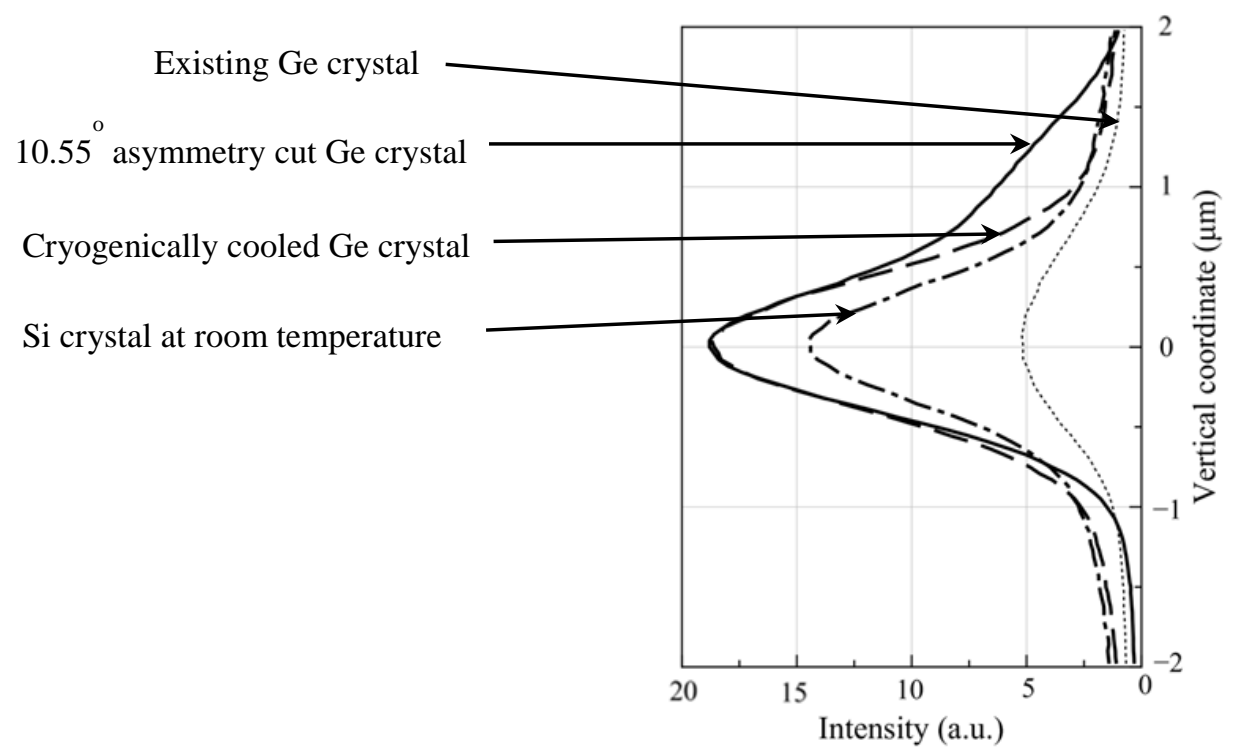

FIG 9. Vertical profiles of the beam focused by the ten beryllium lens system for four different first crystal configurations. Dotted line corresponds to current monochromator; solid line corresponds to the asymmetrically cut germanium monochromator; dashed line corresponds to the cryogenically cooled germanium monochromator; dashed-dotted line corresponds to a silicon monochromator at room temperature.

TABLE I. Focal distances downstream of the monochromator for four different monochromator configurations

\begin{tabular}{ccccc}
\hline \hline Case number & $\begin{array}{c}\text { Existing } \\
\text { system }\end{array}$ & $\begin{array}{c}\text { Asymmetry cut } \\
\text { Ge crystal }\end{array}$ & $\begin{array}{c}\text { Cryogenically } \\
\text { cooled Ge crystal }\end{array}$ & $\begin{array}{c}\text { Si crystal at } \\
\text { room }\end{array}$ \\
\hline Focal distance $(\mathrm{mm})$ & 2398 & 2194 & 2635 & 4427 \\
\hline \hline
\end{tabular}

The presented ray-tracing results are in a good correlation with the analytically estimated focal distances in section 2.

The impact of thermal deformations of the first crystal in a double crystal monochromator on the focal distance of a CRL system downstream of the monochromator is studied. The thermally deformed first crystal acts as a lens creating a virtual source closer to the crystal than the real source at the center of the upstream undulators. The focal distance is thus significantly larger than expected. Since purely geometric changes in the germanium crystal do not improve deformations, three other options to reduce thermal deformations were explored: (1) an asymmetric cut germanium crystal, (2) a cryogenic cooled germanium crystal, and (3) a silicon monochromator. Each has tradeoffs, for example, both the asymmetric-cut 
germanium and symmetrically cut silicon monochromators reduce the spectral bandwidth while cryogenic cooling requires complicated modifications to the crystal system and chamber. These factors will be weighted in the design of an upgrade system. The main point of this study, however, is to demonstrate that deformations that have insignificant impact on beam intensity can have significant impact on focusing due to the well-known lensing effect of a deformed monochromator. An approximate analytical equation to predict the change in focal distance was provided and it was emphasized that the focusing requirements can impose more stringent limits on the thermal deformation of a crystal than throughput and rocking curve requirements because changes in photon energy leads to a moving focus in the former but only a change in intensity in the latter.

\section{ACKNOWLEDGMENTS}

This work is supported in part by the U.S. Department of Energy, Office of Science, under Contract No. DE-AC0206CH11357. The encouragement and cooperation of Prof. F. Mashayek (UIC) are graciously acknowledged.

\section{REFERENCES}

${ }^{1}$ N. Wang, et al., Nuclear Science and Techniques, 21 (2010) 65.

${ }^{2}$ Smither, Rev. Sci. Instrum. 60, 2044 (1989)

${ }^{3}$ Berman, Rev. Sci. Instrum. 66, 2041 (1995))

${ }^{4}$ Chumakov, J. Synchrotron Rad. 11, 132 (2004);

${ }^{5}$ Rutishauser et al., J. Synchrotron Rad. (2013). 20, 300-305

${ }^{6}$ Huang et al., J Synchrotron Rad. 2014 Mar 1; 21(Pt 2): 366-375.

${ }^{7}$ M.R. Howells, et al., Optical Engineering 39 (2000) 2748.

${ }^{8}$ T. Kimura, et al., Proceedings of SPIE 7077, Advances in X-Ray/EUV Optics and Components III (2008) 707709.

${ }^{9}$ A. Snigirev, et al., Journal of Physics: Conference Series 186 (2009) 012073.

${ }^{10}$ B. Cederström, et al., Nature 404 (2000) 951.

${ }^{11}$ R. K. Smither, G. A. Forster, C. A. Kot, T. M. Kuzay, Nuclear Instruments and Methods in Physics Research Section A 266 (1988) 517.

${ }^{12}$ G. Marot, et al., Review of Scientific Instruments 63 (1992) 477.

${ }^{13}$ A. M. Khounsary, Rev. Sci. Instrum. 63 (1992) 461.

${ }^{14}$ S. Brauer, et al., Rev. Sci. Instrum. 66 (1995) 1506.

${ }^{15}$ L. Zhang, M. Sanchez del Rio and G. Monaco, Journal of Physics: Conference Series 425 (2013) 052008. 
$256 \quad{ }^{16}$ R.J. Dejus, et al., ANL/APS/TB-45 (2002).

$257 \quad{ }^{17}$ A.R. Sandy, et al., J. Synchrotron Rad. 6 (1999) 1174.

$258 \quad{ }^{18} \mathrm{X}$-ray Oriented Programs, http://www.esrf.eu/computing/scientific/xop2.1/documentation.html

$259 \quad{ }^{19} \mathrm{P}$. Willmott, An introduction to synchrotron radiation: techniques and applications, 2011

$260 \quad{ }^{20}$ Zemax 13 from Radiant Zemax Corporation, Redmond, Washington

$261 \quad{ }^{21}$ A.M. Khounsary et al., Proceedings of SPIE 3151, High Heat Flux and Synchrotron Radiation Beamlines (1997) 45

$262{ }^{22}$ A. Hedayat, A.M. Khounsary, F. Mashayek, Proceedings of SPIE 8502, Advances in X-Ray/EUV Optics and 263 Components VII (2012) 850200 